\title{
Loss of the $\mathbf{N}$-terminal domain of chlorophyllide $a$ oxygenase induces photodamage during greening of Arabidopsis seedlings Akihiro Yamasato*1,2, Ryouichi Tanaka ${ }^{1}$ and Ayumi Tanaka ${ }^{1}$
} Address: ${ }^{1}$ Institute of Low Temperature Science, Hokkaido University, Sapporo 060-0819, Japan and ${ }^{2}$ Division of Photobiology, National Institute
for Basic Biology, Okazaki 444-8585, Japan

Email: Akihiro Yamasato* - yamasato@nibb.ac.jp; Ryouichi Tanaka - rtanaka@lowtem.hokudai.ac.jp; Ayumi Tanaka - ayumi@pop.lowtem.hokudai.ac.jp

* Corresponding author

Published: 12 June 2008

BMC Plant Biology 2008, 8:64 doi:10.1 I86/147/-2229-8-64
Received: 5 November 2007

Accepted: 12 June 2008

This article is available from: http://www.biomedcentral.com/I47/-2229/8/64

(C) 2008 Yamasato et al; licensee BioMed Central Ltd.

This is an Open Access article distributed under the terms of the Creative Commons Attribution License (http://creativecommons.org/licenses/by/2.0), which permits unrestricted use, distribution, and reproduction in any medium, provided the original work is properly cited.

\begin{abstract}
Background: Chlorophyll $b$ is a major photosynthetic pigment in green plants that is synthesized by chlorophyllide $a$ oxygenase (CAO). The regulation of chlorophyll $b$ biosynthesis is an important determinant for the antenna size of photosystems. Chlorophyll $b$ synthesis is partly regulated on a transcriptional level by the expression of the $C A O$ gene. In addition, the synthesis of chlorophyll $b$ is strictly regulated on a protein level by the stability of the CAO enzyme. CAO consists of three domains, which are sequentially named from the $N$ terminus as the $A, B$ and $C$ domains. The $A$ domain of CAO participates in the regulation of the CAO protein stability.
\end{abstract}

Results: In order to clarify the physiological function of the A domain, we constructed transgenic Arabidopsis (Arabidopsis thaliana) plants which either overexpressed the complete CAO or a truncated version of CAO lacking the A domain. The transgenic plants overexpressing the Adomain-deleted CAO accumulated an excess amount of chlorophyll $b$ during greening. The transgenic plants which lacked the A domain either died or were obviously retarded when they were exposed to continuous light immediately after etiolation. In addition, the loss of the A domain in CAO impaired another step of chlorophyll biosynthesis, namely the conversion of divinylprotochlorophyllide $a$ to monovinyl protochlorophyllide $a$ under dark conditions.

Conclusion: The A domain of CAO regulates the level of CAO, and thus prevents the excess accumulation of chlorophyll $b$. This function of the A domain is especially important during the greening stage of etiolated seedlings. At this stage, the plants are vulnerable to photodamages which could be caused by excessive chlorophyll $b$ accumulation. In addition, de-regulation of the CAO level affects monovinyl-protochlorophyllide biosynthesis in darkness by unknown mechanisms. In conclusion, the A domain of CAO is essential in the control of chlorophyll biosynthesis and in the survival of seedlings during de-etiolation especially under strong illumination.

\section{Background}

Chlorophyll molecules are major components of photosynthesis and play essential roles in harvesting light energy and charge separation. Chlorophylls are actively synthesized during greening and are assembled with various apoproteins to form chlorophyll-protein complexes. Apoproteins of core antenna complexes solely bind chlorophyll $a$, while apoproteins of peripheral antenna com- 
plexes bind both chlorophyll $a$ and chlorophyll $b$ [1]. Since these chlorophyll-protein complexes are stoichiometrically assembled to form functional photosystems, chlorophyll metabolism must be tightly regulated to coordinate with the formation of these complexes. Previous reports have suggested that the regulation of chlorophyll metabolisms is important from the viewpoint of photodamage. Most of the chlorophyll intermediates are photoreactive compounds [2]. If the regulation of 5aminolevulinic acid (ALA), a precursor of tetrapyrrole synthesis, is defective as the result of mutation of a regulation factor, protochlorophyllide is accumulated in excessive amounts under dark conditions [3]. Consequently, this overabundance of protochlorophyllide results in the generation of reactive oxygen species (ROS) and finally causes growth retardation or cell death during greening [3].

At the last step of chlorophyll biosynthesis, chlorophyll $b$ is synthesized from chlorophyll $a$ by chlorophyllide $a$ oxygenase (CAO)[4]. Since an increase in CAO mRNA levels stimulates chlorophyll $b$ synthesis, CAO activity is considered to be partly regulated by $C A O$ gene expression [5-7]. In addition to the regulation on the transcriptional level, chlorophyll $b$ synthesis is also regulated by protein stability. CAO consists of three domains, which are named sequentially from the $\mathrm{N}$ terminus as the $\mathrm{A}, \mathrm{B}$ and $\mathrm{C}$ domains [8]. The $\mathrm{C}$ domain contains binding motifs for a Rieske center and non-heme iron and catalyzes the conversion of chlorophyll $a$ to chlorophyll $b \quad[4,8,9]$. Although the precise function of the $\mathrm{B}$ domain is not known, it is considered to possibly function as a linker between the $\mathrm{A}$ and $\mathrm{C}$ domains [10]. Although the $\mathrm{A}$ domain is not involved in catalytic function, it regulates CAO protein stability $[8,11]$. When the A domain was removed from $\mathrm{CAO}$, and only $\mathrm{BC}$ domains were introduced and overexpressed in Arabidopsis (Arabidopsis thaliana), the protein levels of CAO were drastically increased and chlorophyll $b$ was excessively accumulated [11]. We recently determined that the chloroplast Clp protease plays an important role in CAO protein stability [12]. However, excess chlorophyll $b$ accumulation does not result in a distinct alteration of photosynthetic activity and does not induce photodamage in green leaves [13]. Therefore, at the present time it is still not known why the protein level of CAO is strictly regulated by the A domain.

In order to answer this question, we introduced genes corresponding to either the complete $\mathrm{CAO}$ sequence or $\mathrm{BC}$ domains into Arabidopsis. We subsequently investigated the greening processes of these transgenic plants under various light conditions. Interestingly, transgenic plants expressing only the $\mathrm{BC}$ domains died when the etiolated seedlings were exposed to continuous light. In addition, chlorophyll synthesis was disturbed in these plants during etiolation and greening of seedlings. We discuss the role of the A domain in the regulation of chlorophyll synthesis and chloroplast development.

\section{Results \\ $C A O$ without the $A$ domain caused photodamage during greening}

In order to elucidate the physiological role of the A domain, we examined the light sensitivity of two types of transgenic plants that we named $\mathrm{tGBC} c h$ and $\mathrm{tGABC} c h$ in the ch1-1 mutant background. The ch1-1 mutant contains a deletion in the $C A O$ gene and does not contain chlorophyll $b$ [14]. The tGBCch lines overexpress a chimeric transgene containing the GFP gene and the CAO gene lacking the A-domain coding sequence [11]. In contrast, the $\mathrm{tGABC}$ ch lines overexpress another chimeric transgene encoding GFP and the full-length CAO protein [11]. Both types of transgenic plants were grown under different light conditions and their phenotypes were compared to those of wild type and the ch1-1 mutant. When the seeds were germinated and grown under low light conditions, all four strains grew well, although the ch1-1 exhibited a pale green phenotype (Fig. 1A). When etiolated seedlings were transferred to low light conditions, most of the tGBCch plants were bleached and the growth of the tGBCch seedlings was retarded (Fig. 1C). When exposed to high light conditions, no etiolated and green seedlings of tGBCch survived (Fig. 1B and 1D). On the contrary, seedlings from the other three strains developed normally and grew under low and high light conditions (Fig. 1B, C and 1D). These results confirmed that the overexpression of the $C A O$ gene lacking the A domain resulted in photodamage to Arabidopsis seedlings.

GFP-CAO fusion transgenes were highly overexpressed in the etiolated and greening seedlings of all transgenic tGA$\mathrm{BC} c h$ and $\mathrm{tGBC} c h$ plant lines (Fig. 2A). The expression levels of the transgenes, which correspond to GFP-ABC or GFP-BC, were higher than that of full-length CAO in wild type seedlings (Fig. 2A). The tGBCch transgenic lines accumulated a large amount of GFP-BC protein (a fusion of GFP and B-C domains of CAO) in both etiolated and green seedlings (Fig. 2B and 2C). The GFP-BC proteins localized to etioplasts and chloroplasts (Fig. 3). In contrast, the full-length CAO (A-B-C domains) and the GFPfull-length CAO (a fusion of GFP and A-B-C domains of $\mathrm{CAO}$ ) proteins were under a detectable level by immunoblotting in both etiolated and greening seedlings (Fig. 2B). It was evident that the full-length CAO and the GFPfull-length CAO proteins accumulated to certain levels in the green leaves because chlorophyll $b$ was actively synthesized in the transgenic plants [11]. However, the protein levels of $\mathrm{CAO}$ in the seedlings were too low for detection by immunoblot analysis. These data suggest that the low accumulation level of CAO is sufficient to 
A

(Low light)

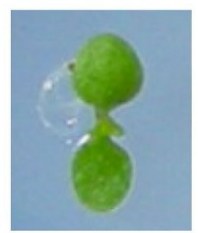

Wild type

B

(High light)

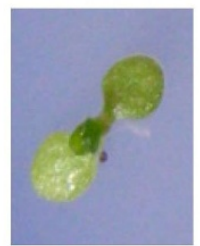

Wild type

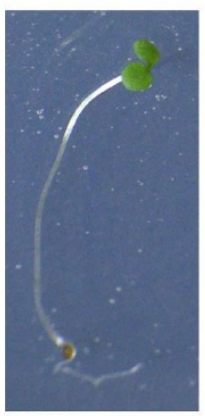

Wild type

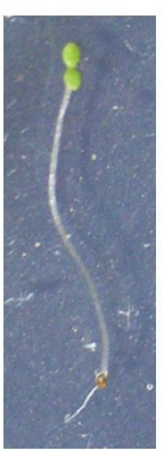

Wild type

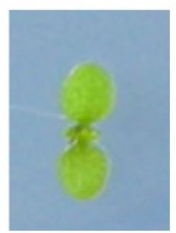

ch1-1

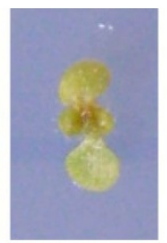

ch1-1

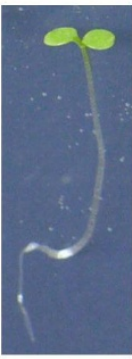

ch1-1



ch1-1

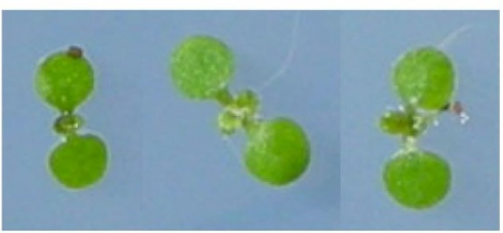

tGABCch1, 2, 3

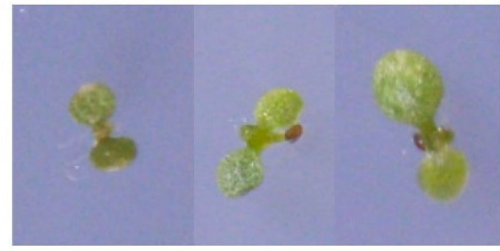

tGABCch1, 2, 3

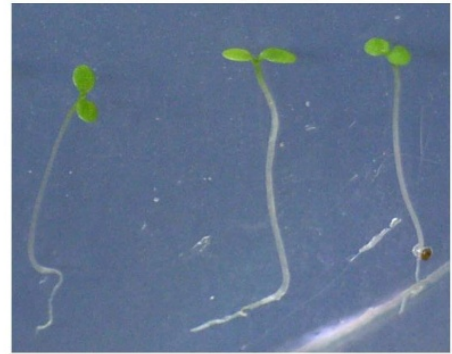

tGABCch1, 2, 3



tGABC $\operatorname{ch} 1,2,3$

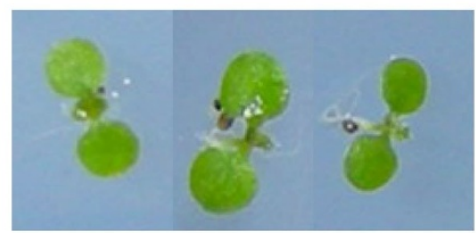

tGBCch1, 2, 3

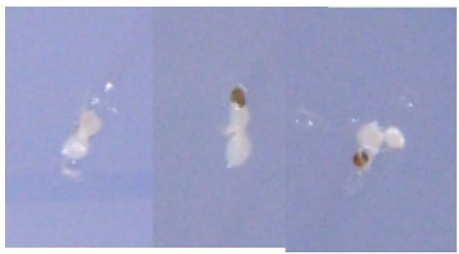

tGBC ch1, 2, 3

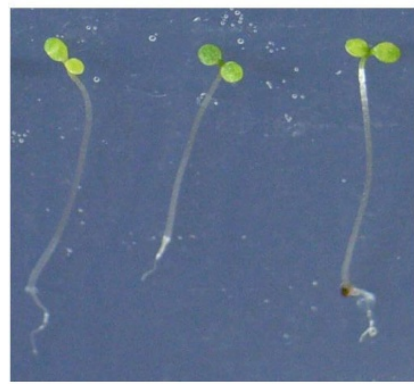

tGBC ch1, 2, 3

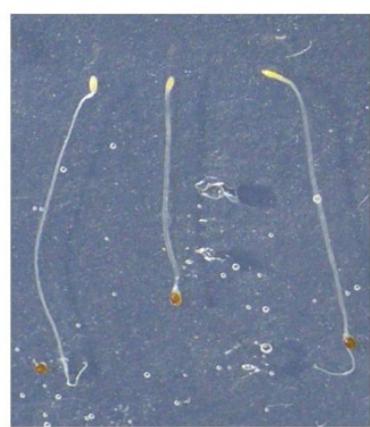

tGBC ch1, 2, 3

Figure I

Light sensitivities of the Arabidopsis seedlings. A and B: Arabidopsis seedlings were germinated and grown continuously under low $\left(70 \mu \mathrm{E} \mathrm{m}^{-2} \mathrm{~s}^{-1}\right)$ or high light $\left(300 \mu \mathrm{E} \mathrm{m} \mathrm{m}^{-2} \mathrm{~s}^{-1}\right)$ conditions for 7 days. $C$ and $\mathrm{D}$ : Etiolated seedlings were grown for 4 days and transferred to low or high light conditions for an additional 3 days. Numbers after transgenic plants (for example, tGBCchI, 2, 3) indicate independent transgenic lines for each type of transgenic plants. 
A

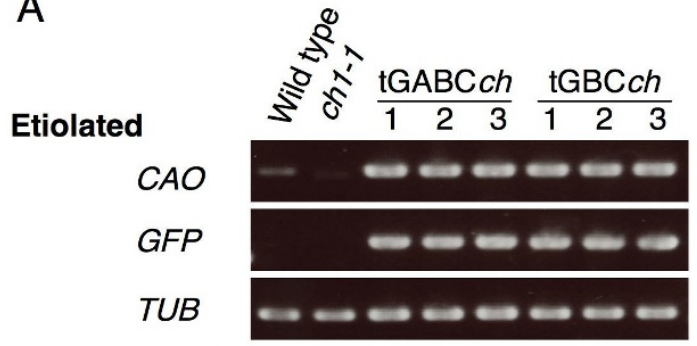

Greening (24 h)

$C A O$

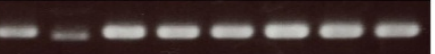

GFP

TUB

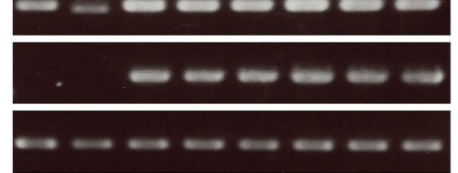

B

Etiolated

CAO

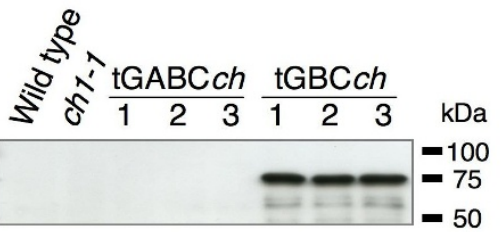

GFP

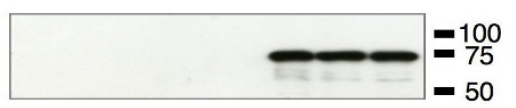

Greening (24 h)

CAO

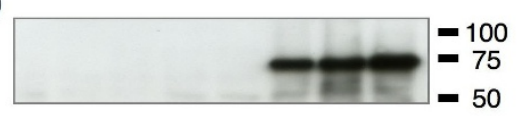

GFP

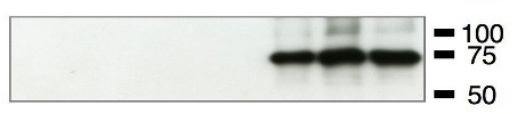

C

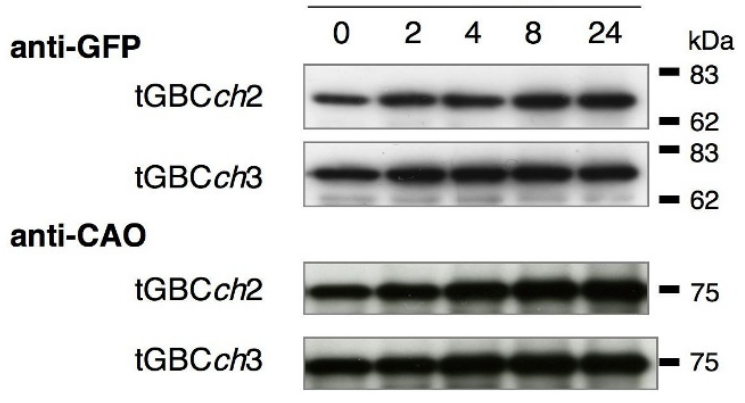

Figure 2

Expression levels of CAO in the etiolated and greening seedlings. A: The expression levels of internal CAO gene and GFP-CAO transgenes in 4-day-old etiolated seedlings and greening seedlings were determined by semi-quantitative PCR. Expression levels from the tubulin (TUB2) gene were monitored as a quantitative control. The internal CAO expression level increased in wild type and $c h l-I$ seedlings during greening. The $C A O$ transcript of $c h l-I$ has a $3 I$ bp deletion and it was not detected in the tGABCch and tGBCch plants. B: The accumulation levels of the CAO proteins and GFP-CAO fusion proteins in 4-day-old etiolated seedlings and in greening seedlings were determined. The estimated molecular size of the full-length CAO, GFP-ABC and GFP-BC proteins without transit peptide were 56,83 and $70 \mathrm{kDa}$, C: The accumulation levels of the GFP-BC proteins in 4day-old etiolated tGBCch seedlings during a $24 \mathrm{~h}$ greening period were determined. The full-length CAO or GFP-CAO fusion proteins were not detected in wild type, chl-I and tGABCch seedlings (data not shown). 


\section{Greening (h)}

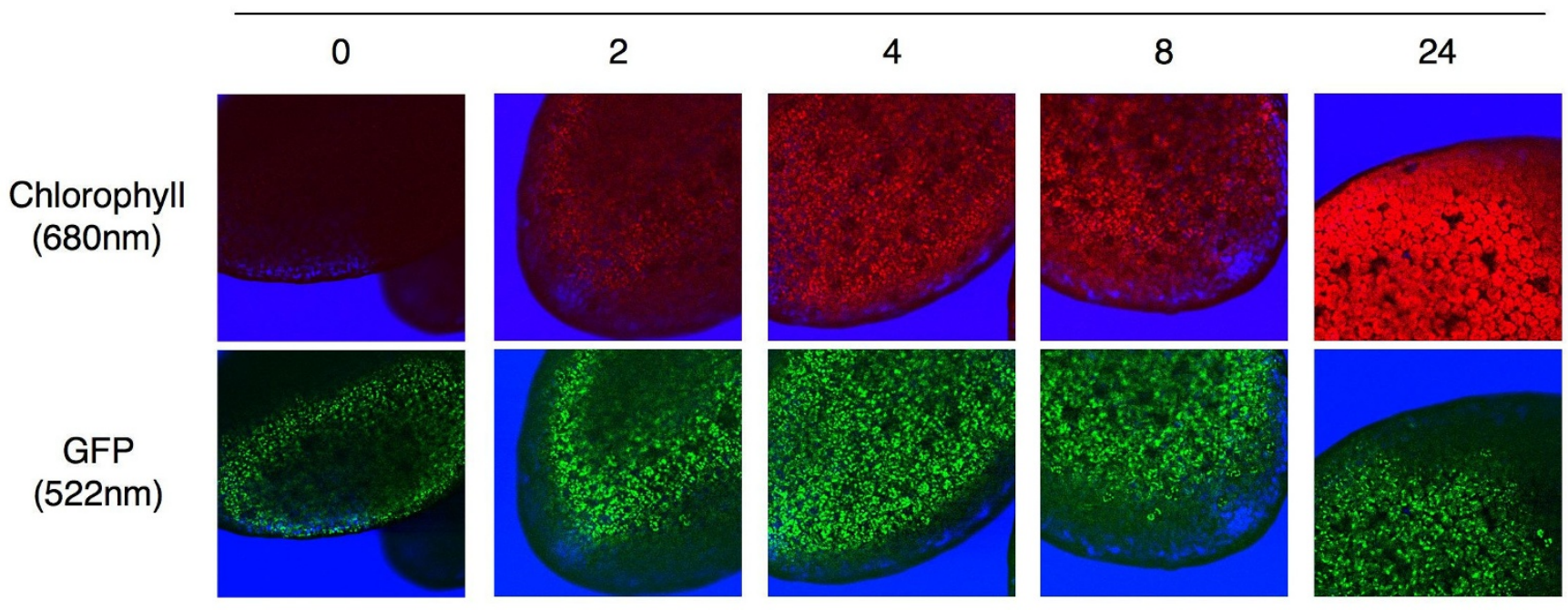

tGBCch3

\section{Figure 3}

Accumulation of chlorophyll and the GFP-BC protein in the tGBCch seedlings during greening. The fluorescence images of the Arabidopsis cotyledons during greening for $24 \mathrm{~h}$ were observed with confocal microscopy. The GFP-CAO protein and the plastids during greening are indicated by green and red fluorescence, respectively. The green fluorescence of GFP$\mathrm{BC}$ protein was detected only within plastids during greening.

synthesize chlorophyll $b$. We should note that the excess accumulation of the fusion protein in the GFP-BC overexpressing plants was not due to the transcriptional or translational activation in these plants [11]. It was most likely that the lack of the A domain impaired the degradation of CAO by Clp protease [12].

As shown in Figs. 1 and 2, our transgenic lines did not show any obvious phenotypical differences between the lines. In the subsequent experiments described within this study, we also examined all transgenic lines shown in Figs. 1 and 2 . All of the lines produced results that were nearly identical.

Next, we examined the accumulation of chlorophyll when etiolated seedlings were transferred to continuous light conditions. In comparison to wild type, chlorophyll levels were low in tGBCch and ch1-1 but slightly higher in tGABCch (Fig. 4). In good accordance with chlorophyll accumulation, Lhcb protein levels were higher in tGABCch and lower in tGBCch compared to wild type (Fig. 5). The chlorophyll $a / b$ ratio was very high soon after the onset of illumination and decreased gradually during the greening in wild type (Fig. 6). In contrast, the chlorophyll $a / b$ ratio was already low after illumination and gradually increased during greening in tGABCch and tGBCch (Fig. $6)$. In tGBCch, the chlorophyll $a / b$ ratio was extremely low. Fig. 7 shows the HPLC profiles of chlorophyll that accumulated after 5 min of illumination in wild type and two types of transgenic plants. Under this condition, most of the chlorophyll molecules were derived from protochlorophyllide $a$ that pre-accumulated during the dark condition. The wild type seedlings accumulated chlorophyll $a$ but not chlorophyll $b$ (Fig. 7). It should be noted that the small peak at $25 \mathrm{~min}$ in wild type was not chlorophyll $b$ because the spectrum showed different peaks (data not shown). In contrast, the tGBCch only accumulated chlorophyll $b$, suggesting that chlorophyll $a$ (or chlorophyllide $a$ ) in tGBCch was immediately converted to chlorophyll $b$ (or chlorophyllide $b$ ) after the photoconversion of protochlorophyllide $a$ into chlorophyllide $a$ (Fig. 7). Both chlorophyll $a$ and chlorophyll $b$ accumulated in tGA$\mathrm{BC} c h$ and the level of chlorophyll $a$ was nearly equivalent to the levels of wild type (Fig. 7).

Interestingly, we found that the fully-greened leaves of tGBCch accumulated a significant amount of 7 hydroxymethyl chlorophyll, which is an intermediate molecule in the conversion between chlorophyll $\mathrm{b}$ and chlorophyll a (Fig. 8). The unusually high level of 7- 


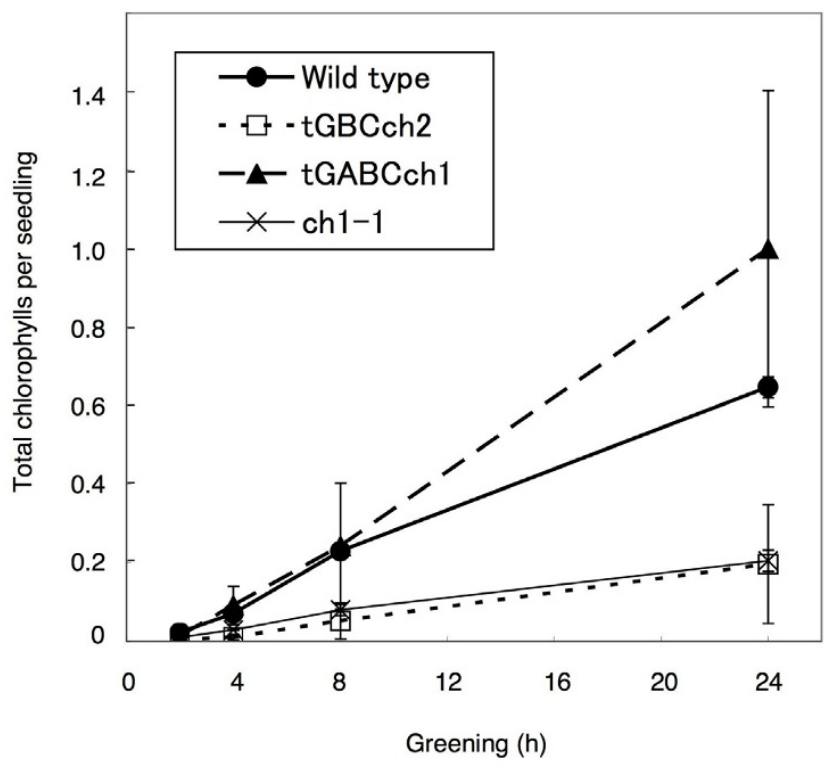

Figure 4

Chlorophyll levels in the seedlings during greening. Etiolated seedlings were grown for 4 days under dark conditions. The etiolated seedlings were then exposed to low light conditions and were harvested during greening at the indicated times. The total amount of chlorophyll per seedling was determined with HPLC analysis. The error bars represent standard deviations $(n=4)$.

hydroxymethyl chlorophyll suggests that the excess GFP$\mathrm{BC}$ proteins or the excess chlorophyll $\mathrm{b}$ might activate the conversion between chlorophyll $\mathrm{a}$ and $\mathrm{b}$ [15].

\section{Loss of the A domain results in a perturbation of chlorophyll metabolism}

The tGBCch seedlings accumulated a low level of chlorophyll during the greening process (Fig. 4). These data are consistent with the observation that the amount of protochlorophyllide, which is an end product of chlorophyll synthesis in etiolated seedlings, decreased in tGBCch transgenic plants (Table 1 ). When plants were fed with ALA, similar trends were observed between wild type and the transgenic plants (Table 1). These results might suggest that certain enzymatic steps between ALA and protochlorophyllide formation were disturbed and that this disturbance limited the chlorophyll synthesis in the tGBCch seedlings during greening.

In our next line of investigation, we examined the re-accumulation of protochlorophyllide in the dark after a brief illumination as a means to analyze the kinetics of protochlorophyllide synthesis in the seedlings. Under these conditions, protochlorophyllide levels were similar in wild type, tGBCch and tGABCch during the first day of

\section{Greening $(24 \mathrm{~h})$}
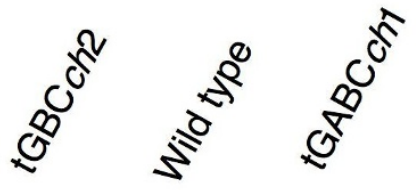

\author{
Lhcb
}

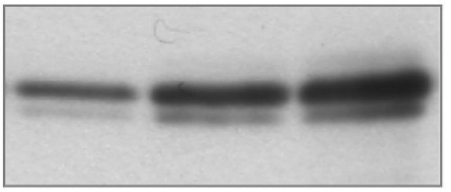

\section{Figure 5}

Lhcb protein levels in the greening seedlings. Etiolated seedlings were first grown for 4 days under dark conditions and were subsequently exposed to low light conditions for $24 \mathrm{~h}$. The accumulation levels of the Lhcb proteins in the greening seedling were determined by immunoblotting using anti-Lhcb antibody. Lhcb protein levels in the $\mathrm{ch} /$-I seedlings after $24 \mathrm{~h}$ of greening were nearly equivalent to those from tGBCch (data not shown).

darkness (Table 2). However, the levels decreased in the following 2-day period of darkness only in tGBCch (Table 2). Although the etiolated tGBCch seedlings seemed to synthesize protochlorophyllide normally, the pigment appeared to be broken down during dark condition by unknown mechanisms. Alternatively, It is also possible that divinyl-protochlorophyllide $a$ may inhibit an earlier step of chlorophyll biosynthesis in a feedback manner.

In order to investigate the chlorophyll $b$ synthetic pathway in tGBCch, we compared the compositions of the chlorophyll intermediates in wild type, tGBCch and tGABCch. In spite of the accumulation of $\mathrm{CAO}$ in tGBCch, protochlorophyllide $b$ was not detected in etiolated seedlings (Fig. 9 ). These data indicate that CAO is unable to catalyze the conversion of protochlorophyllide $a$ to protochlorophyllide $b$ in Arabidopsis. Interestingly, a significant difference was observed in the accumulation ratios of divinyl-protochlorophyllide $a$ to total protochlorophyllide $a$ among wild type, tGBCch and tGABCch (Fig. 9 and Table 1). The etiolated seedlings of wild type and tGABCch predominately accumulated monovinyl-protochlorophyllide $a$ (Table 1). Conversely, monovinyl-protochlorophyllide $a$ was accumulated to very low levels in tGBCch (Table 1). This observation indicates that the loss of the A domain from CAO resulted in an inactivation of 3, 8-divinyl protochlorophyllide a 8-vinyl reductase [16].

\section{Discussion}

The A domain protects cells against photodamage during greening

We previously reported on the light sensitivity of transgenic Arabidopsis plants overexpressing prokaryotic CAO 
Table I: Total protochlorophyllide accumulation in etiolated seedlings.

\begin{tabular}{ccccc}
\hline Strains & \multicolumn{2}{c}{ Total protochlorophyllide/etiolated seedling (pmole) } & & Divinyl-protochlorophyllide/total protochlorophyllide (molar ratio) \\
\cline { 2 - 3 } & -ALA & +ALA & -ALA \\
\hline Wild type & $0.78 \pm 0.02$ & $8.09 \pm 1.98$ & & $0.37 \pm 0.03$ \\
tGBCch3 & $0.33 \pm 0.01$ & $2.56 \pm 0.56$ & & $0.80 \pm 0.05$ \\
tGABCch3 & $1.01 \pm 0.09$ & $6.29 \pm 2.08$ & & $0.32 \pm 0.03$ \\
\hline
\end{tabular}

The seedlings were grown for 6 days under dark conditions with or without ALA $\left(0.5 \mathrm{mg} \mathrm{ml}^{-1}\right)$ on wet filter paper. Seedlings were subsequently harvested under dark conditions and their extracts were analyzed for their protochlorophyllide composition by HPLC analysis $(n=5)$.

which does not have the A domain. These transgenic plants accumulated much more chlorophyll $b$ than tGBCch plants [13]. In this previous report, we concluded that the excess accumulation of chlorophyll $b$ did not cause photodamage in the fully greened leaves of the transgenic plants [13]. However, in this study, we found that most of the etiolated tGBCch seedlings died during greening not only under high light conditions, but also under low light conditions. Without the presence of chlorophyll $a$, chlorophyll $b$ would not be incorporated into apoproteins. As a consequence, the free chlorophyll $b$ might generate ROS [2]. In contrast to tGBCch, transgenic plants overexpressing the full-length CAO accumulated both chlorophyll $a$ and chlorophyll $b$ immediately after the onset of illumina-

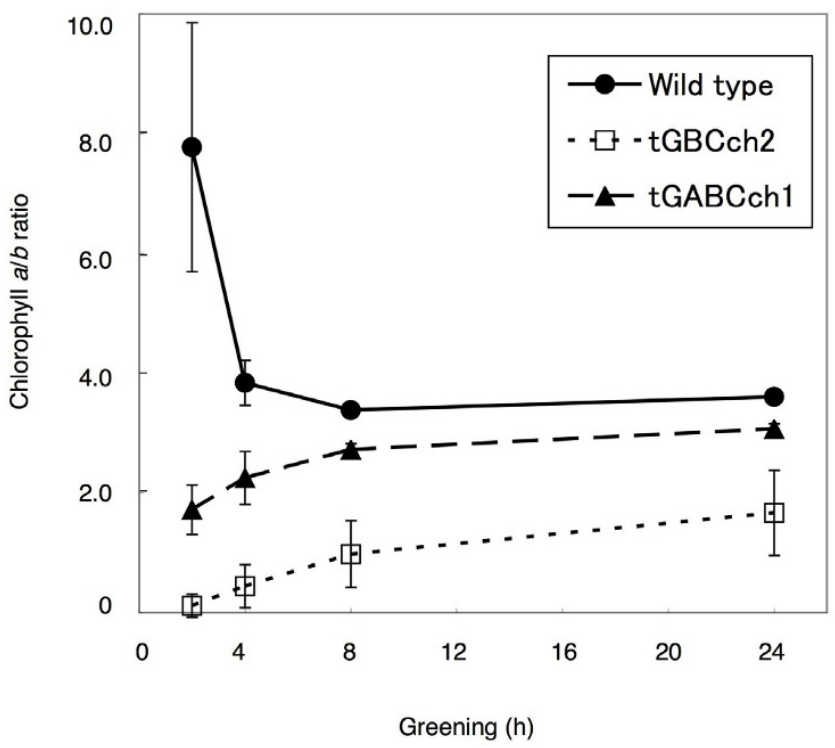

Figure 6

Molar ratio of the chlorophyll $a$ and $b$ contents in the seedlings during greening. The etiolated seedlings were first grown for 4 days under dark conditions and were subsequently exposed to low light. The compositions of chlorophyll $a$ and $b$ were calculated from chromatographic peak profiles at $650 \mathrm{~nm}$ with HPLC analysis. The error bars represent standard deviations $(n=4)$. tion. Unlike tGBCch plants, the full-length-CAO overexpression lines did not exhibit photodamage. It is possible that chlorophyll $b$ is assembled with apoproteins in the presence of chlorophyll $a$. As a result, the generation of ROS might have been suppressed. Collectively, these results indicate that the A domain is essential in preventing photodamages that could be caused by excess chlorophyll $b$ accumulation during greening. The etiolated seedlings of the transgenic Arabidopsis overexpressing prokaryotic $\mathrm{CAO}$ might cause obvious photodamage.

An unusually high level of 7-hydroxymethyl chlorophyll $a$, an intermediate molecule in the conversion between chlorophyll $b$ to chlorophyll $a$, accumulated in the green leaves of tGBCch (Fig. 8). This observation might indicate that excess chlorophyll $b$ is reconverted to chlorophyll $a$ by the chlorophyll cycle [15]. It is not likely that 7 hydroxymethyl chlorophyll was formed in the course of the chlorophyll $a$ to chlorophyll $b$ conversion. Since CAO catalyzes the two-step reaction from chlorophyll $a$ to chlorophyll $b$ by itself, the intermediate molecule, 7hydroxymethyl chlorophyll, is supposed to be associated with the CAO enzyme and is not released during the reaction [14]. In our study, 7-hydroxymethyl chlorophyll was not found just after the start of the illumination of etiolated seedlings, although chlorophyll $b$ was actively synthesized (Fig. 7). Therefore, it would be reasonable to assume that 7-hydroxymethyl chlorophyll was predominantly formed in the course of the chlorophyll $b$ to chlorophyll $a$ conversion.

\section{Accumulation of GFP-BC protein interferes with the regulation of chlorophyll biosynthesis}

In comparison to wild type plants, chlorophyll accumulation during greening was suppressed in tGBCch seedlings, even under low light conditions. It is possible that this suppression might be due to the oxidative stress generated by free chlorophyll $b$. The overabundance of protochlorophyllide generates ROS, which causes growth retardation or cell death during greening in the flu mutant [3]. Recently, we found that the chlorophyll biosynthesis pathway is a target of oxidative stress [17]. However, all of the phenotypes of tGBCch could not be explained by oxi- 


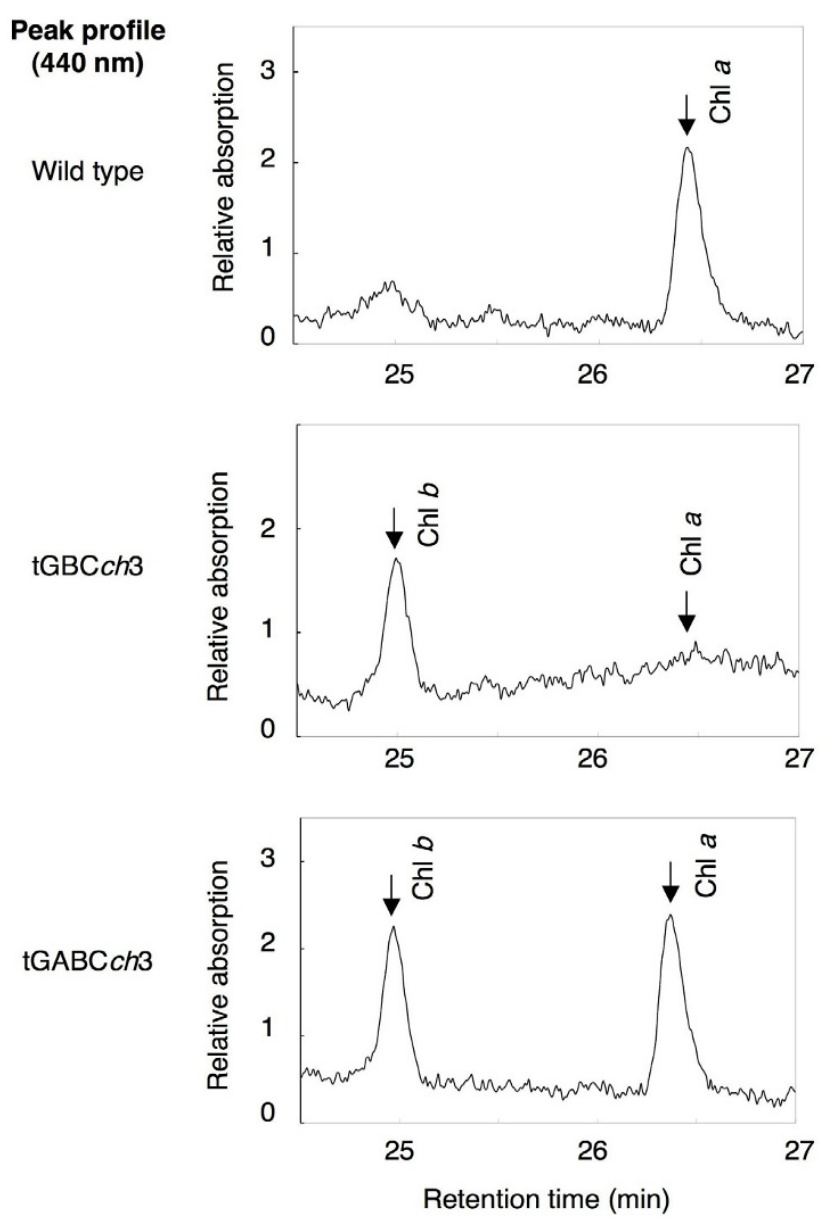

Figure 7

Chlorophyll accumulation in etiolated Arabidopsis seedlings after light treatments. Chlorophyll accumulation after exposure to low light for $5 \mathrm{~min}$ in 6-day-old etiolated seedlings. The chlorophyll $a$ and $b$ extracted from the seedlings were separated by HPLC analysis. $\mathrm{Chl} a$, chlorophyll $a$; Chl $b$, chlorophyll $b$.

dative stress alone. For example, the level of protochlorophyllide $a$ in etiolated tissues of tGBCch was lower than that of wild type. Since these plants were not exposed to light, the involvement of ROS in the inhibition of protochlorophyllide accumulation can be excluded. We also observed an increase in the ratio of divinyl-protochlorophyllide to the total protochlorophyllide in tGBCch transgenic plants. These data indicate that the accumulation of the $\mathrm{BC}$ domains of CAO inhibits the activity of 3, 8-divinyl protochlorophyllide $a$ 8-vinyl reductase. On the contrary, overexpression of the GFP-ABC protein enhanced chlorophyll accumulation. These phenomena are very interesting in terms of biological regulation since both CAO and truncated CAO affected not only chlorophyll $b$ synthesis but also other chlorophyll metabolic steps.
These results are reasonable because some enzymes, which are related to chlorophyll biosynthesis, are reported to form complexes or interact with other enzymes. For example, glutamyl-tRNA reductase and glutamate-1-semialdehyde aminotransferase form a complex which enables an efficient substrate trafficking between two enzymes [18]. In addition, $\mathrm{BchH}$, one of the three subunits of $\mathrm{Mg}$ chelatase of Rhodobacter capsulatus, accelerated the Mg-protoporphyrin IX monomethyl transferase activity [19]. If CAO does indeed interact with other enzymes, the accumulation of truncated CAO could potentially interfere with the activity of the target enzymes.

In addition, the loss of the A domain in CAO impaired the conversion of divinyl-protochlorophyllide $a$ to monovinyl protochlorophyllide $a$, and obviously reduced total protochlorophyllide $a$ level under dark conditions. A possible scenario for the reduction of protochlorophyllide accumulation in the tGBCch seedlings is that the excessively-accumulated CAO in these seedlings interfered with the enzyme that catalyzes the formation of monovinylprotochlorophyllide $a$ from divinyl-protochlorophyllide $a$, the latter of which may inhibit an earlier step of chlorophyll biosynthesis in a feedback manner. Further studies are necessary and warranted to clarify this question.

\section{CAO does not catalyze the conversion of protochlorophyllide a to protochlorophyllide b}

When etiolated seedlings of wild type are exposed to continuous light, chlorophyll $a$ immediately accumulates after the onset of illumination. Subsequent to illumination, chlorophyll $a$ and $b$ levels gradually increase after several hours of a lag phase. Conversely, in tGBCch plants, chlorophyll $b$ accumulated but chlorophyll $a$ was not detected after 5 min of illumination (Fig. 7). Considering that protochlorophyllide $b$ was not found in etiolated seedlings, these data indicate that chlorophyll $a$ was immediately converted to chlorophyll $b$ in tGBCch seedlings during the early phase of greening (Fig. 9).

Several research groups report that protochlorophyllide $b$ does not exist in etioplasts $[20,21]$. Contrary to these reports, another group claimed that the protochlorophyllide $b$ could be detected in etiolated seedlings when they use diethylpyrrocarbonate-containing acetone for the extraction of pigments [22]. It was also proposed that protochlorophyllide $b$ is synthesized via a pathway that is independent from the CAO protein [23]. In addition, a cyanobacteria transformant with the CAO and $L h c b$ transgenes, accumulated protochlorophyllide $b$ [24]. This observation indicates that $\mathrm{CAO}$ is capable of converting protochlorophyllide $a$ to protochlorophyllide $b$. In this study, we used $100 \%$ acetone as an extraction solvent for chlorophyll intermediates in order to avoid the artificial 

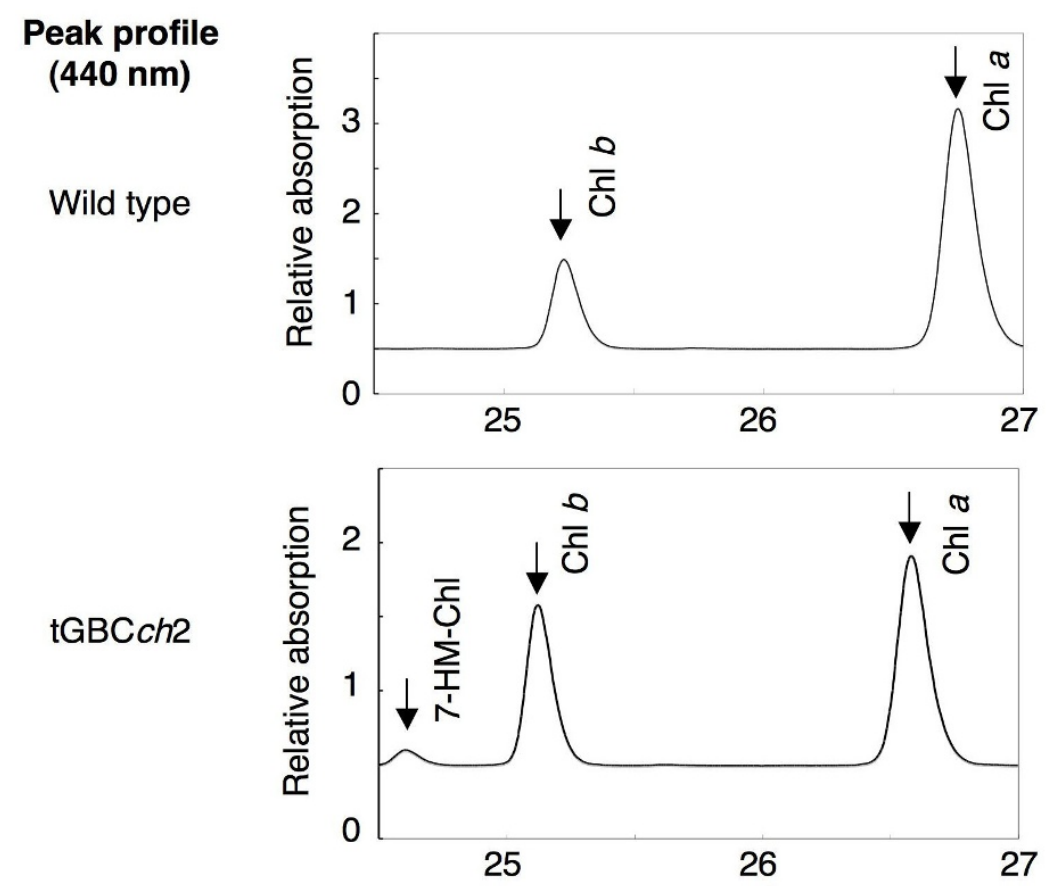

tGABC ch1
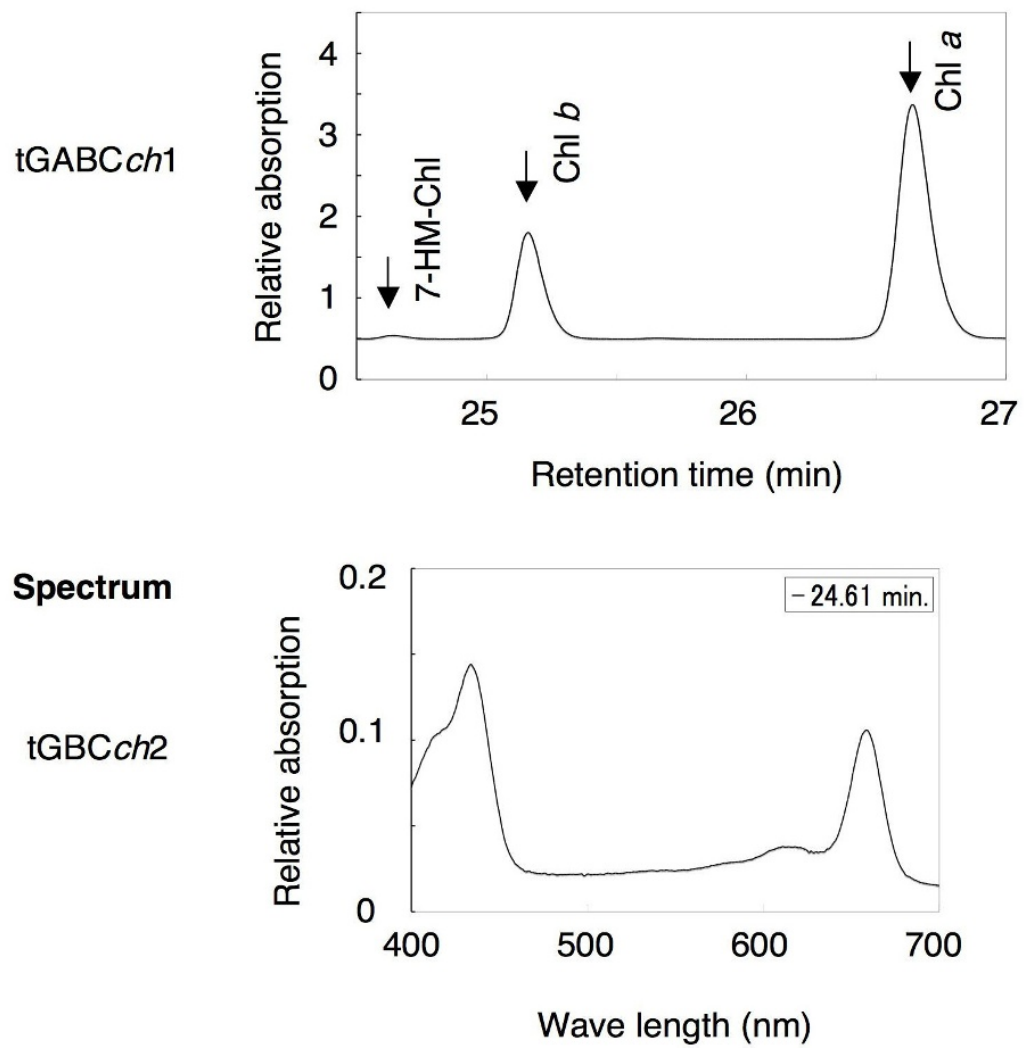

Figure 8

Chlorophyll accumulation in greened seedlings. Chlorophyll accumulation in fully greened seedlings that were grown for 8 days under low light conditions. The chlorophyll $a, b$ and 7-hydroxymethyl chlorophyll extracted from the seedlings were separated by HPLC analysis. The spectrum of a peak that was eluted at $24.6 \mathrm{~min}$ in the extract from the tGBCch leaves is shown. Chl $a$, chlorophyll $a$; Chl $b$, chlorophyll $b$; 7-HM-Chl, 7-hydroxymethyl chlorophyll. 
Table 2: Total protochlorophyllide accumulation in etiolated seedlings during dark incubation.

\begin{tabular}{cccc}
\hline Strains & \multicolumn{2}{c}{ Total protochlorophyllide/etiolated seedling (pmole) } \\
\cline { 2 - 4 } & \multicolumn{3}{c}{ Dark incubation after light treatment } \\
\cline { 2 - 4 } & 0 day & I day & 3 days \\
Wild type & n.d. & $0.70 \pm 0.01$ & $0.77 \pm 0.16$ \\
tGBCch3 & n.d. & $0.49 \pm 0.01$ & $0.25 \pm 0.02$ \\
tGABCch3 & n.d. & $0.68 \pm 0.13$ & $1.28 \pm 0.01$
\end{tabular}

Six-day-old etiolated seedlings were exposed to low light for $5 \mathrm{~min}$ and were returned to dark conditions. The seedlings after a $I$ or 3 day additional dark incubation period were subsequently harvested under dark conditions. Their extracts were analyzed for their protochlorophyllide composition by HPLC analysis $(n=3)$. The n.d. abbreviation indicates "not detected".

formation of chlorophyll derivatives and to maximize our extraction of the chlorophyll intermediates from seedlings [21]. Consequently, we did not detect any protochloro- phyllide $b$ in etiolated tGBCch seedlings, even though a large amount of the CAO protein accumulated. These results indicate that protochlorophyllide $b$ is not a major intermediate product for chlorophyll $b$ synthesis in planta.

\section{Conclusion}

Taken together, our results demonstrated that excessive accumulation of chlorophyll $b$ is harmful to plants. This deleterious effect likely disturbs the proper synthesis of chlorophyll intermediates and the accumulation of photosynthetic proteins. Therefore, we conclude that the regulation of the CAO protein by the A domain is essential for the survival of etiolated seedlings under light conditions. Since the A domain functions to maintain proper chlorophyll $b$ levels throughout the development of plants [11], it would be reasonable to assume that the regulation of the CAO protein level is essential in various aspects of plant development and acclimation to light conditions. Further studies on the regulatory mechanism
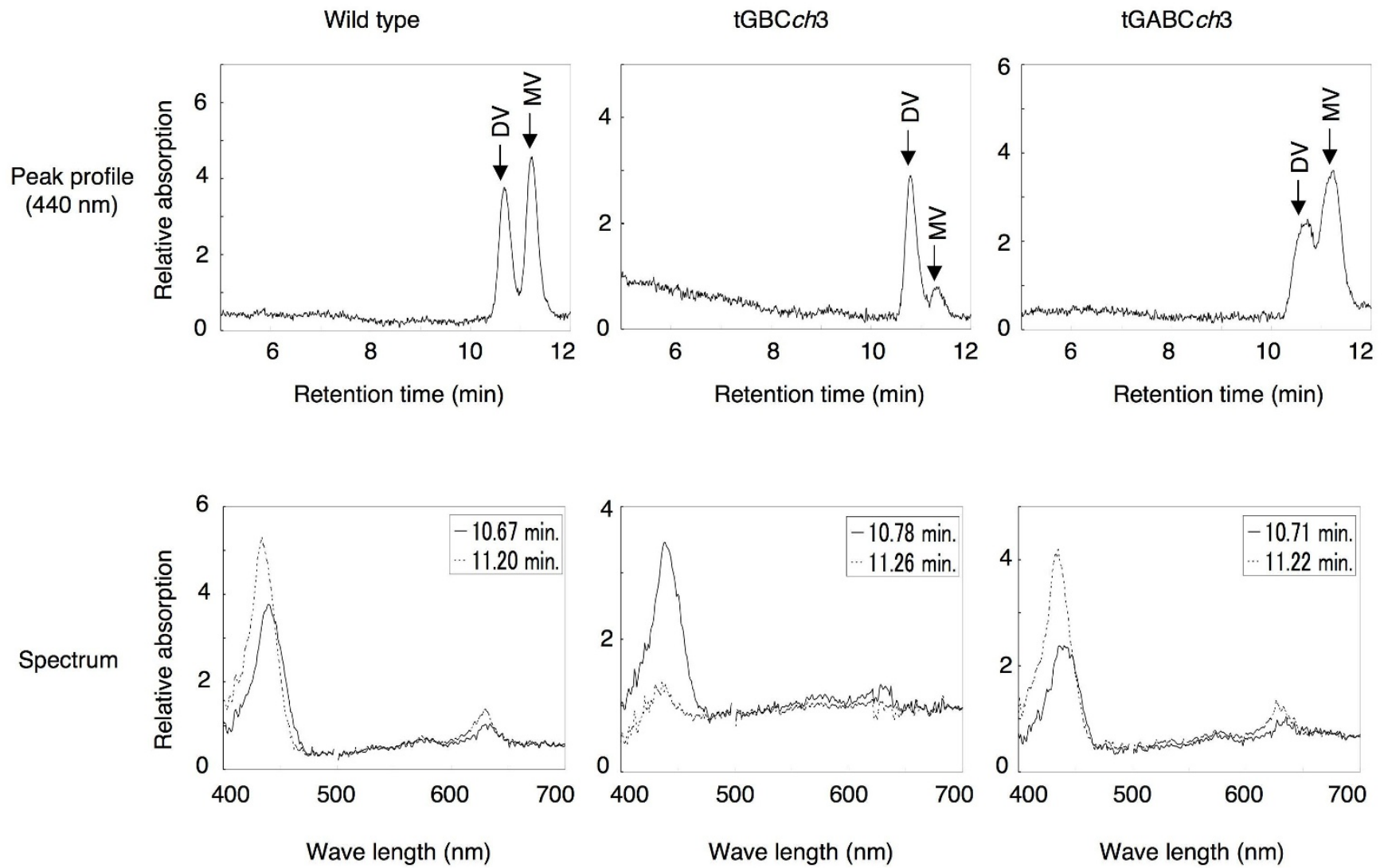

Figure 9

Chromatographic peaks and spectra of divinyl- and monovinyl-protochlorophyllide $a$ in etiolated seedlings. Seedlings were grown for 6 days under dark conditions on wet filter paper without ALA. The seedlings were harvested under dark conditions and their extracts were analyzed for their protochlorophyllide composition by HPLC. DV, divinyl-protochlorophyllide $a$; MV, monovinyl-protochlorophyllide $a$. 
of CAO may elucidate the physiological roles of this mechanism throughout the life cycle of plants.

\section{Methods}

\section{Plant materials and growth conditions}

The Arabidopsis CAO protein consists of three domains, which are respectively named: A (V37-L170), B (P171G200) and C (A201-G537) domains [8]. In this study, we used Arabidopsis thaliana Columbia wild type and the ch11 mutant which contains a deletion in the CAO gene [14]. In addition, we also used two types of transgenic plants overexpressing chimeric fusions of the $\mathrm{CAO}$ protein and the GFP. The two Arabidopsis transformants, tGBCch and tGABCch, expressed fusion transgenes corresponding to transit peptide-GFP-B-C domains and transit peptideGFP-A-B-C domains in the ch1-1 background, respectively [11]. Three homozygous lines for each transgenic plant tGBCch and tGABCch, were used in this study. All of the transgenic plants were isolated independently. Arabidopsis seedlings were grown at $22^{\circ} \mathrm{C}$ on agar plates $(0.7 \%[\mathrm{w} /$ v]) containing $1 / 2$ diluted Murashige-Skoog medium. Plants were grown under low light $\left(70 \mu \mathrm{E} \mathrm{m}^{-2} \mathrm{~s}^{-1}\right.$ from fluorescent bulbs) or high light $\left(300 \mu \mathrm{E} \mathrm{m}^{-2} \mathrm{~s}^{-1}\right.$ from xenon light bulbs [3.6 kW] equipped with ND filters, TGE-2S3, Tabai, Osaka, Japan) conditions.

\section{Reverse transcriptase $P C R$}

Total RNA was extracted using an RNeasy Plant Mini Kit (Qiagen, Hilden, Germany) from 200 etiolated seedlings which were grown under dark conditions for 4 days. RNA was extracted from etiolated seedlings after a period of greening for 24 hours. One $\mu \mathrm{g}$ of isolated RNA was reverse-transcribed into cDNA using a SuperScript III Kit (Invitrogen, Carlsbad, CA). PCR was performed to determine gene expression level using the synthesized firststranded cDNA sample in a $25 \mu \mathrm{l}$ reaction. PCR conditions were as follows: $96^{\circ} \mathrm{C}(2 \mathrm{~min}) ; 25$ cycles of $96^{\circ} \mathrm{C}$ $(30 \mathrm{sec}), 55^{\circ} \mathrm{C}(30 \mathrm{~min}), 72^{\circ} \mathrm{C}(1 \mathrm{~min})$. The amount of cDNA template for the PCR was normalized by $\beta$-tubulin (TUB2) gene expression level. The primers used for RTPCR were as follows: For TUB2, 5'-CTC AAG AGG TTC TCA GCA GTA-3' and 5'-TCA CCT TCT TCA TCC GCA GTT-3'; For CAO, 5'-AAC GAG GGA CGT ATT CAA TGT CCG-3' and 5'-AGA AGA AGG TAA ACA GAC ATG G-3'; For GFP, 5'-ATG GTG AGC AAG GGC GAG G-3' and 5'TTA CTT GTA CAG CTC GTC CA-3'.

\section{Immunoblotting}

Ten seedlings were homogenized with $100 \mu$ l of extraction buffer ( 50 mM Tris [pH 6.8], 2 mM EDTA, 10\% [w/v] glycerol, $2 \%[\mathrm{w} / \mathrm{v}]$ SDS, $6 \%[\mathrm{v} / \mathrm{v}] 2$-mercaptoethanol). The isolated supernatants $(25 \mu \mathrm{l})$ were subsequently subjected to SDS-PAGE. The resolved proteins were blotted onto a hybond-P membrane (GE Healthcare, Buckinghamshire, UK). A 1/5000-diluted anti-GFP (Invitrogen) antibody, a 1/2000-diluted anti-CAO antibody [11] and a 1/5000diluted anti-Lhcb [6] rabbit antibody were used to detect specific proteins as previously described [11]. Cross-reactive protein bands were developed using anti-rabbit IgG that was linked to horseradish peroxidase (GE Healthcare). An ECL plus western blotting analysis kit was use for the chemiluminescent detection of antigens (GE Healthcare).

\section{Confocal microscopy}

Fluorescence images were recorded on an Axioplan fluorescence microscope $(\times 20$ objective lens, Carl Zeiss, Jena, Germany) which was integrated in an MRC 1024 confocal laser-scanning microscopic system (Bio-rad laboratories, Hercules, CA). Samples were excited by an argon laser (25 $\mathrm{mW}$ ) at $488 \mathrm{~nm}$ and the GFP and chlorophyll fluorescence were recorded at 522 and $680 \mathrm{~nm}$, respectively. The images were processed with the Adobe Photoshop 4.0 software (Adobe, San Joze, CA).

\section{HPLC analysis}

Chlorophylls were initially extracted from the seedlings with $100 \%$ acetone and the extracts were subsequently diluted to $80 \%$ acetone with water. Diluted extracts were then subjected to HPLC analysis (Shim-pack CLC-ODS column, $6.0 \times 150 \mathrm{~mm}$; Shimadzu, Kyoto, Japan) using methanol as the elution agent at a flow rate of $1.7 \mathrm{ml} \mathrm{min}^{-}$ 1 . The compositions of chlorophyll species were calculated from chromatographic peak profiles at $650 \mathrm{~nm}$ [6].

Protochlorophyllides extracted from the seedlings were analyzed by HPLC on a Symmetry C8 column $(4.6 \times 150$ $\mathrm{mm}$; Waters Corporation, MA, USA) according to the previously described method of Zapata et al. [25]. Elution profiles and the spectra of the eluted pigments were recorded continuously in the range of 400 to $700 \mathrm{~nm}$ by SPD-M $10 A_{A V}$ (Shimadzu). The compositions of protochlorophyllide were calculated from chromatographic peak profiles at $440 \mathrm{~nm}$ [26].

\section{Authors' contributions}

AY participated in the design of the study and the writing of the manuscript, and carried out all of the experimental studies. RT participated in the design of the study, HPLC and physiological analyses and the writing of the manuscript. AT participated in the design of the study and the writing of the manuscript. All authors read and approved the final manuscript.

\section{Acknowledgements}

This work was supported by a Grant-in-Aid for Creative Scientific Research (No. 17GS03I4 to AT) and Grants-in-Aid for Scientific Research (No. 18770025 to AY; No. 68700307 to RT) from the Ministry of Education, Culture, Sports, Science and Technology of Japan. We would like to thank Drs. R. Hellens and P. Mullineaux (John Innes Centre, UK) for kindly providing the pGreen II plasmids. We would also like to thank Dr. Y. Niwa 
(Division of Food Science, University of Shizuoka, Japan) for providing us with the sGFP (S65T) plasmid.

\section{References}

I. Green BR, Durnford DG: The chlorophyll-carotenoid proteins of oxygenic photosynthesis. Annu Rev Plant Physiol Plant Mol Biol | 996, 47:685-7|4.

2. Vavilin DV, Vermaas WFJ: Regulation of the tetrapyrrole biosynthetic pathway leading to heme and chlorophyll in plants and cyanobacteria. Physiol Plant 2002, I I 5:9-24.

3. Meskauskiene R, Nater M, Goslings D, Kessler F, Camp Ro-d, Apel K: FLU: A negative regulator of chlorophyll biosynthesis in Arabidopsis thaliana. Proc Natl Acad Sci USA 200।, 98: | 2826-|283|.

4. Tanaka A, Ito H, Tanaka R, Tanaka N, Yoshida K, Okada K: Chlorophyll $a$ oxygenase (CAO) is involved in chlorophyll $b$ formation from chlorophyll a. Proc Natl Acad Sci USA 1998, 95: I27|9-12723.

5. Espineda CE, Linford AS, Devine D, Brusslan JA: The AtCAO gene, encoding chlorophyll $a$ oxygenase, is required for chlorophyll b synthesis in Arabidopsis thaliana. Proc Natl Acad Sci USA 1999, 96: I0507-105II.

6. Tanaka R, Koshino Y, Sawa S, Ishiguro S, Okada K, Tanaka A: Overexpression of chlorophyllide $a$ oxygenase (CAO) enlarges the antenna size of photosystem II in Arabidopsis thaliana. Plant J 200I, 26:365-373.

7. Harper $A L$, von Gesjen SE, Linford AS, Peterson MP, Faircloth RS, Thissen MM, Brusslan JA: Chlorophyllide a oxygenase mRNA and protein levels correlate with the chlorophyll alb ratio in Arabidopsis thaliana. Photosynth Res 2004, 79: I49-I 59.

8. Nagata N, Satoh S, Tanaka R, Tanaka A: Domain structures of chlorophyllide $a$ oxygenase of green plants and Prochlorothrix hollandica in relation to catalytic functions. Planta 2004, 2 1 8: 1019-1025.

9. Tomitani A, Okada K, Miyashita H, Matthijs HCP, Ohno T, Tanaka A: Chlorophyll $b$ and phycobilins in the common ancestor of cyanobacteria and chloroplasts. Nature 1999, 400:159-162.

10. Sakuraba Y, Yamasato A, Tanaka R, Tanaka A: Functional analysis of N-terminal domains of Arabidopsis chlorophyllide a oxygenase. Plant Physiol Biochem 2007, 45:740-749.

II. Yamasato A, Nagata N, Tanaka R, Tanaka A: The N-terminal domain of chlorophyllide $a$ oxygenase confers protein instability in response to chlorophyll $b$ accumulation in Arabidopsis. Plant Cell 2005, I 7: I 585- I597.

12. Nakagawara E, Sakuraba Y, Yamasato A, Tanaka R, Tanaka A: Clp protease controls chlorophyll $b$ synthesis by regulating the level of chlorophyllide a oxygenase. Plant J 2007, 49:800-809.

13. Hirashima M, Satoh S, Tanaka R, Tanaka A: Pigment shuffling in antenna systems achieved by expressing prokaryotic chlorophyllide a oxygenase in Arabidopsis. J Biol Chem 2006, 28I:|5385-15393.

14. Oster U, Tanaka R, Tanaka A, Rüdiger W: Cloning and functional expression of the gene encoding the key enzyme for chlorophyll $b$ biosynthesis (CAO) from Arabidopsis thaliana. Plant J 2000, 2 I:305-3 I0.

15. Ohtsuka T, Ito H, Tanaka A: Conversion of Chlorophyll b to Chlorophyll $a$ and the assembly of chlorophyll with apoproteins by isolated chloroplasts. Plant Physiol 1997, I I 3: I 37-I 47.

16. Nagata N, Tanaka R, Satoh S, Tanaka A: Identification of a vinyl reductase gene for chlorophyll synthesis in Arabidopsis thaliana and implications for the evolution of Prochlorococcus species. Plant Cell 2005, I 7:233-240.

17. Aarti PD, Tanaka R, Tanaka A: Effects of oxidative stress on chlorophyll biosynthesis in cucumber (Cucumis sativus) cotyledons. Physiol Plant 2006, I 28: I86-197.

18. Nogaj LA, Beale SI: Physical and kinetic interactions between glutamyl-tRNA reductase and glutamate-I-semialdehyde aminotransferase of Chlamydomonas reinhardtii. J Biol Chem 2005, 280:24301-24307.

19. Gibson LC, Willows RD, Kannangara CG, von Wettstein D, Hunter $\mathrm{CN}$ : Magnesium-protoporphyrin chelatase of Rhodobacter sphaeroides: reconstitution of activity by combining the products of the bchH, -I, and -D genes expressed in Escherichia coli. Proc Natl Acad Sci USA 1995, 92:194I-1944.
20. Scheumann V, Klement H, Helfrich M, Oster U, Schoch S, Rudiger W: Protochlorophyllide $b$ does not occur in barley etioplasts. FEBS Lett 1999, 445:445-448.

21. Kolossov VL, Rebeiz CA: Chloroplast Biogenesis 88 Protochlorophyllide $b$ occurs in green but not in etiolated plants. $J$ Biol Chem 2003, 278:49675-49678.

22. Reinbothe S, Pollmann S, Reinbothe C: In situ conversion of protochlorophyllide $b$ to protochlorophyllide $a$ in barley. Evidence for a novel role of 7 -formyl reductase in the prolamellar body of etioplasts. J Biol Chem 2003, 278:800-806.

23. Reinbothe S, Quigley F, Gray J, Schemenewitz A, Reinbothe C: Identification of plastid envelope proteins required for import of protochlorophyllide oxidoreductase $\mathbf{A}$ into the chloroplast of barley. Proc Natl Acad Sci USA 2004, I 0 I:2 1 97-2202.

24. Xu H, Vavilin D, Vermaas $W$ : The presence of chlorophyll $\boldsymbol{b}$ in Synechocystis sp. PCC 6803 disturbs tetrapyrrole biosynthesis and enhances chlorophyll degradation. J Biol Chem 2002, 277:42726-42732.

25. Zapata M, Rodríguez F, Garrido JL: Separation of chlorophylls and carotenoids from marine phytoplankton: A new HPLC method using a reversed phase $C 8$ column and pyridine containing mobile phases. Mar Ecol Prog Ser 2000, I 95:29-45.

26. Nagata N, Tanaka $R$, Tanaka A: The major route for chlorophyll synthesis includes [3,8-divinyl]-chlorophyllide $a$ reduction in Arabidopsis thaliana. Plant Cell Physiol 2007, 48: I803-I808.

Publish with Bio Med Central and every scientist can read your work free of charge

"BioMed Central will be the most significant development for disseminating the results of biomedical research in our lifetime. "

Sir Paul Nurse, Cancer Research UK

Your research papers will be:

- available free of charge to the entire biomedical community

- peer reviewed and published immediately upon acceptance

- cited in PubMed and archived on PubMed Central

- yours - you keep the copyright 